Subnanomet er - resol ut $i$ on fr equency modul at $i$ on at om c force microscopy in I i qui d for bi ol ogi cal appl i cat i ons

\begin{tabular}{|l|l|}
\hline 著者 & Fukuma Takeshi \\
\hline $\begin{array}{l}\mathrm{j} \text { our nal or } \\
\text { publ i cat i on ti t l e }\end{array}$ & Japanese Journal of Appl i ed Physi cs \\
\hline vol une & 48 \\
\hline nunber & 8 \\
\hline page r ange & 08J A01 \\
\hline year & 2009- 08 01 \\
\hline URL & ht t p: //hdl . handl e. net /2297/24291 \\
\hline
\end{tabular}




\title{
Subnanometer-Resolution Frequency Modulation Atomic Force Microscopy in Liquid for Biological Applications
}

\author{
Takeshi FukumA ${ }^{1,2 *}$ \\ ${ }^{1}$ Frontier Science Organization, Kanazawa University, Kakuma-machi, Kanazawa 920-1192, Japan \\ ${ }^{2}$ PRESTO, Japan Science and Technology Agency, 4-1-9 Honcho, Kawaguchi 332-0012, Japan
}

The spatial resolution and force sensitivity of frequency modulation atomic force microscopy (FM$\mathrm{AFM}$ ) in liquid have been dramatically improved in the last a few years. It is now possible to image individual atoms and molecules at a solid/liquid interface with a subnanometer-scale resolution and a piconewton-order loading force. This capability enabled the direct visualization of hydration layers and mobile ions on a lipid bilayer and $\beta$-strands constituting an amyloid fibril. These striking results highlighted the significant potential of FM-AFM in biological research. Here, I summarize the technological innovation that brought about this progress and review biological applications of FM-AFM in liquid.

\section{Introduction}

Frequency modulation atomic force microscopy (FM-AFM) was introduced by Albrecht et al. in 1991 for operating dynamic-mode atomic force microscopy (AFM) in ultrahigh vacuum (UHV) environments. ${ }^{1)}$ Operation in a UHV environment allows us to investigate atomically clean and well-defined surfaces of various materials. ${ }^{2}$ ) The force sensitivity of FM-AFM is greatly enhanced by operating FM-AFM in a vacuum chamber owing to the high $\mathrm{Q}$ factor of cantilever resonance in vacuum. These factors, together with advancements in instrumentation, made it possible to achieve true atomic resolution in UHV on various surfaces including semiconductors, ${ }^{3,4)}$ metals, ${ }^{5,6)}$ metal oxides, ${ }^{7)}$ alkali halides ${ }^{8)}$ and organic materials. ${ }^{9)}$

A decade after the first demonstration of true atomic resolution by FM-AFM, ${ }^{3,4)}$ it has become routine to carry out atomic-scale imaging by FM-AFM in UHV. Various FM-AFM-based techniques for surface property measurements have also been developed, including Kelvinprobe force microscopy, ${ }^{10)}$ scanning capacitance force microscopy, ${ }^{11)}$ and magnetic exchange force microscopy. ${ }^{12}$ ) Precise and stable control of the tip position using the feedforward drift compensation technique has also made it possible to manipulate a single atom at room temperature. ${ }^{13)}$ The use of a quartz tuning fork sensor and small oscillation amplitude has enabled subatomic-scale imaging, ${ }^{14)}$ which encouraged scanning tunneling microscopy (STM) users to introduce a simultaneous STM/FM-AFM setup into their systems. ${ }^{15)}$

In contrast to the advancement of FM-AFM in UHV, its applications in air and liquid have

\footnotetext{
${ }^{*}$ E-mail address: fukuma@staff.kanazawa-u.ac.jp
} 
been limited. There are several reasons for this. The original motivation for using FM-AFM was to operate dynamic-mode AFM in vacuum for enhancing the force sensitivity and investigating atomically clean surfaces in a well-defined environment. ${ }^{1)}$ Thus, it has not been clear whether the force sensitivity required for true atomic-resolution imaging can be obtained, and whether a cantilever can be stably oscillated with a self-excitation circuit in low-Q environments (i.e., in air and liquid). Particularly in liquid, contact-mode AFM has been widely used for visualizing molecular-scale arrangements of membrane proteins. ${ }^{16)}$ Therefore, unless clear advantages of FM-AFM over contact-mode AFM are experimentally demonstrated, it will be difficult to encourage users of contact-mode AFM or researchers in nanobioscience to employ FM-AFM in their experiments.

The challenge of operating FM-AFM in liquid was initiated by Jarvis et al. in 2000.17) Their work was motivated by an interest in probing the oscillatory solvation force presented by the solvation layers formed at a solid/liquid interface. As an oscillatory solvation force has a large force gradient, it was necessary to use FM-AFM with a stiff cantilever to avoid instability known as "jump-to-contact". At this stage, it was still difficult to operate the tip-sample distance feedback regulation on a frequency shift $(\Delta f)$ signal, so that a dissipation signal was used instead. In 2002, Kobayashi et al. succeeded in operating the tip-sample distance feedback regulation on a $\Delta f$ signal in liquid using a custom-built phase-locked loop (PLL) circuit, and presented an FM-AFM image of $\mathrm{Au}(111)$ surface obtained in water. ${ }^{18,19)}$ Subsequently, Okajima et al. also developed a custom-built PLL circuit and compared the amplitude and frequency changes of a self-oscillating cantilever near the surface. ${ }^{20,21)}$ Although these pioneering works demonstrated that FM-AFM can be operated in liquid, the spatial resolution of FM-AFM in liquid at this stage was not as high as that obtained by contact-mode AFM or amplitude-modulation AFM (AM-AFM).

The situation was dramatically changed in 2005. Fukuma et al. developed a multienvironment FM-AFM with true subnanometer resolution imaging capability in vacuum, air and liquid. ${ }^{22)}$ The first true molecular- and atomic-resolution FM-AFM images obtained in liquid were presented by imaging a polydiacetylene single crystal ${ }^{23)}$ and a muscovite mica ${ }^{24}$ in water, respectively. This dramatic progress in the FM-AFM performance in low-Q environments was brought about by three major improvements in its instrumentation and operating conditions. These key factors are described in detail in $\S 2$. In 2006, Fukuma et al. developed a custom-built liquid-environment FM-AFM with improved performance. ${ }^{25)}$ The developed FM-AFM was applied to subnanometer-scale investigations of biological systems, ${ }^{26-28)}$ which highlighted the distinctive advantages of FM-AFM over conventional AFM techniques in liquid. These biological applications are reviewed in $\S 3$. While these recent experiments demonstrated the applicability of FM-AFM to biological research, they also revealed the existence of remaining issues that have yet to be solved to make this technique more practical and useful. 
These issues and their possible solutions are discussed in $\S 4$.

\section{Instrumentation}

The spatial resolution and force sensitivity of FM-AFM in liquid were dramatically improved in $2005,{ }^{22}$ ) leading to the first demonstration of true atomic resolution imaging by FM-AFM in liquid. ${ }^{24}$ ) There are three major differences in instrumentation and operating conditions between the high-resolution liquid-environment $\mathrm{FM}_{-} \mathrm{AFM}^{22,25)}$ and a typical liquidenvironment AFM. In this section, these key features of the liquid-environment FM-AFM are described.

\subsection{Cantilever stiffness}

The typical spring constant $(k)$ of a cantilever used in a typical liquid-environment AFM is in the range of $0.01-1 \mathrm{~N} / \mathrm{m}$ while that in the high-resolution liquid-environment $\mathrm{FM}-\mathrm{AFM}^{22,25}$ ) is in the range of $20-40 \mathrm{~N} / \mathrm{m}$. The high cantilever stiffness allows stable and precise control of the vertical tip position at atomic-scale contact and noncontact regimes. This capability is essential for true atomic-resolution imaging and other applications such as atom manipulation. ${ }^{13)}$

From the equipartition theorem, the root-mean-square (rms) amplitude of cantilever thermal Brownian vibration $\delta z_{B}$ is given by ${ }^{1)}$

$$
\delta z_{B}=\sqrt{\frac{k_{B} T}{k}}
$$

where $k_{B}$ and $T$ are Boltzmann's constant and the absolute temperature, respectively. For the cantilevers with $k=0.4$ and $40 \mathrm{~N} / \mathrm{m}$, the values for $\delta z_{B}$ are respectively 100 and $10 \mathrm{pm}$. The typical corrugation height of atomic- and molecular-scale features in FM-AFM images is in the range of $10-100 \mathrm{pm}^{2)}$ Thus, the accuracy of control over the vertical tip position required for true atomic-resolution imaging is approximately $10 \mathrm{pm}$. To satisfy this condition, it is necessary to use a relatively stiff cantilever (typically $k>20 \mathrm{~N} / \mathrm{m}$ ).

Note that it is possible to reduce the thermal vibration amplitude by making gentle contact between a tip and a surface such as in contact-mode AFM. However, this results in a loss of control over the vertical tip position at the atomic-scale contact/noncontact regimes and gives rise to a large lateral friction force during imaging. These issues make it difficult to use contact-mode AFM for true atomic-resolution imaging and nondestructive imaging of isolated biomolecules.

A high stiffness cantilever is also required for preventing instabilities known as "jump-tocontact". As the cantilever approaches a surface, the force gradient gradually increases. Once the force gradient exceeds $k$, the cantilever suddenly jumps to contact the surface. Thus, the 
Table I. Typical parameters for a stiff cantilever used in FM-AFM and a soft cantilever used in conventional AFM in liquid.

\begin{tabular}{lcccccc}
\hline & $k$ & $f_{0}$ & $Q$ & $\begin{array}{c}\delta z_{B} \\
(\mathrm{~N} / \mathrm{m})\end{array}$ & $\begin{array}{c}\left|F_{c}\right|_{\min }(\mathrm{pN}) \\
(\mathrm{kHz})\end{array}$ & $\begin{array}{c}n_{z B}\left(f_{0}\right) \\
(\mathrm{pm})\end{array}$ \\
\hline Typical stiff cantilever & 30 & 130 & 8 & 12 & 3.9 & $74 \mathrm{~Hz})$ \\
Typical soft cantilever & 0.1 & 5 & 1.5 & 200 & 2.7 & 2813 \\
\hline
\end{tabular}

criterion for preventing jump-to-contact in contact-mode AFM is given by

$$
k>\left|\frac{\partial F_{\mathrm{ts}}}{\partial z}\right|_{\max },
$$

where $F_{\mathrm{ts}}$ is the tip-sample interaction force.

In the case of dynamic-mode AFMs such as AM-AFM and FM-AFM, jump-to-contact can be prevented by satisfying a condition given by either eq. (2) or

$$
k A>\left|F_{\mathrm{ts}}\right|_{\max }
$$

where $A$ denotes the cantilever oscillation amplitude. Therefore, jump-to-contact can be prevented using a cantilever with a high $k$ or oscillating a cantilever with a large $A$. However, as is discussed later, a small $A$ is desirable for obtaining high spatial resolution. Thus, to perform atomic-scale imaging without instabilities, a stiff cantilever (typically $k>20 \mathrm{~N} / \mathrm{m}$ ) oscillated with a small $A$ should be used.

The minimum detectable conservative tip-sample interaction force $\left(\left|F_{c}\right|_{\min }\right)$ in FM-AFM with the small amplitude approximation is given by ${ }^{29)}$

$$
\left|F_{c}\right|_{\text {min }}=\sqrt{\frac{4 k k_{B} T B}{\pi f_{0} Q}},
$$

where $B, f_{0}$, and $Q$ are the measurement bandwidth, cantilever resonance frequency, and cantilever $\mathrm{Q}$ factor, respectively. This equation shows that the use of a cantilever with a high $k$ results in a reduction of force sensitivity. In practice, this effect is not necessarily significant. A stiff cantilever tends to have smaller dimensions, higher $f_{0}$, and higher $Q$ than a soft cantilever, which partially compensates for the reduction of force sensitivity due to the high $k$. For the typical stiff and soft cantilevers shown in Table I, the values for $\left|F_{c}\right|_{\text {min }}$ $(B=100 \mathrm{~Hz})$ are 3.9 and $2.7 \mathrm{pN}$, respectively. Namely, the typical stiff cantilever has a comparable force sensitivity to the typical soft cantilever but has a much smaller thermal vibration amplitude and higher stability.

\subsection{Cantilever oscillation amplitude}

The typical value for $A$ in conventional liquid-environment AFM is in the range of 5-

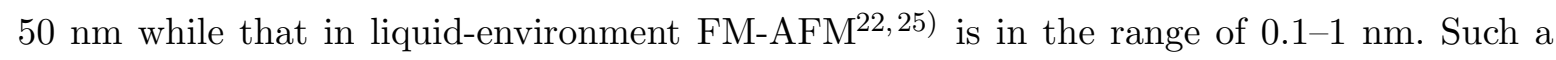


small amplitude operation of FM-AFM is known to enhance the sensitivity to short-range interaction forces ${ }^{30)}$ and thereby improves spatial resolution in FM-AFM imaging. ${ }^{14)}$

$F_{\text {ts }}$ includes long-range forces (e.g., electrostatic and van der Waals forces) and shortrange forces (e.g., chemical interaction and solvation force). To achieve true (i.e., nonaveraged) atomic resolution in AFM, the short-range interaction between the tip front atom and the surface atom or molecule must predominantly contribute to the feedback signal for the tipsample distance regulation (i.e., $z$ in contact-mode AFM, $A$ in AM-AFM, and $\Delta f$ in FMAFM). With a smaller oscillation amplitude in dynamic-mode AFM, a tip front atom can stay in the short-range interaction regime for a longer time in an oscillation period. In the short-range interaction regime, the influence of the long-range interaction force on the force gradient is negligible compared with that of the short-range interaction force. Therefore, the use of a small amplitude enhances sensitivity to the short-range interaction force and reduces sensitivity to the long-range interaction force, leading to a higher spatial resolution.

On the basis of this idea, Giessibl et al. calculated the optimal $A$ for high-resolution FMAFM imaging and reported that it is on the order of the decay length of the force component to be detected. ${ }^{30)}$ For short-range forces that are responsible for atomic-scale contrast formation, the length is typically less than $0.5 \mathrm{~nm}$, which accounts for the high spatial resolution obtained by FM-AFM using a small amplitude (typically $A=0.1-1 \mathrm{~nm}$ ).

In a vacuum environment, cantilever oscillation with such a small amplitude requires the use of a very stiff cantilever (e.g., $1800 \mathrm{~N} / \mathrm{m}$ ) to avoid jump-to-contact. ${ }^{31}$ ) In liquid environments, the long-range forces, such as van der Waals and electrostatic forces, are greatly reduced by the existence of solvent molecules. Thus, a cantilever with a spring constant of $10-40 \mathrm{~N} / \mathrm{m}$ can be used without causing instabilities during imaging even with an $A$ of less than $1 \mathrm{~nm}$. This advantage partially compensates for the disadvantage of having a low $\mathrm{Q}$ factor in liquid. This is one of the major reasons that the spatial resolution obtained in liquid is comparable to that obtained in UHV in spite of the large difference in Q factors obtained in vacuum and liquid.

\subsection{Cantilever deflection sensor}

A cantilever deflection signal contains two major noise components: noise arising from the thermal Brownian vibration of a cantilever and noise arising from the deflection sensor itself. The power spectral density (PSD) of the deflection noise arising from the cantilever deflection sensor $\left(n_{z s}\right)$ in typical liquid-environment AFM is in the range of $100-1,000 \mathrm{fm} / \sqrt{\mathrm{Hz}},{ }^{2)}$ while that in liquid-environment $\mathrm{FM}_{-} \mathrm{AFM}^{22,25)}$ is in the range of $4-40 \mathrm{fm} / \sqrt{\mathrm{Hz}}$. As FM-AFM utilizes a deflection signal to produce a cantilever excitation signal as well as to detect $\Delta f$, the low-noise cantilever deflection signal is essential for stable cantilever self-oscillation with a small oscillation amplitude $(A<1 \mathrm{~nm})$ during high-resolution FM-AFM imaging.

The PSD of the deflection noise arising from the thermal Brownian vibration of a cantilever 
$\left(n_{z B}\right)$ is given by ${ }^{1)}$

$$
n_{z B}=\sqrt{\frac{2 k_{B} T}{\pi f_{0} k Q} \frac{1}{\left[1-\left(f / f_{0}\right)^{2}\right]^{2}+\left[f /\left(f_{0} Q\right)\right]^{2}}},
$$

while $n_{z s}$ is typically white noise in the frequency range relevant to dynamic-mode AFM. In dynamic-mode AFM, only the noise components in the frequency range from $f_{0}-B$ to $f_{0}+B$ influence FM-AFM performance. As $B$ is typically less than $1 \mathrm{kHz}$, the deflection noise in the frequency range is almost constant in liquid and is given by

$$
n_{z B}\left(f_{0}\right)=\sqrt{\frac{2 k_{B} T Q}{\pi f_{0} k}} .
$$

Therefore, to achieve the theoretically limited noise performance given by eq. (4), $n_{z s}$ must be reduced to a value sufficiently smaller than $n_{z B}\left(f_{0}\right)$. For typical soft and stiff cantilevers with the parameters shown in Table I, the values of $n_{z B}\left(f_{0}\right)$ are 2.8 and $74 \mathrm{fm} / \sqrt{\mathrm{Hz}}$, respectively. As shown here, a stiffer cantilever has a lower thermal noise peak and hence requires a lower noise level of the deflection sensor to achieve the theoretically limited noise performance.

The total PSD of a cantilever deflection signal $\left(n_{z}\right)$ is given by

$$
n_{z}=\sqrt{n_{z B}^{2}+n_{z s}^{2}} .
$$

From the ratio between $n_{z}\left(f_{0}\right)$ and $n_{z B}\left(f_{0}\right)$, we can estimate the increase in the noise owing to $n_{z s}$ as shown in Fig. 1. To suppress the noise increase to a value of less than $10 \%, n_{z s}$ should be less than $34 \mathrm{fm} / \sqrt{\mathrm{Hz}}$ for the typical stiff cantilever with the parameters shown in Table I. The $n_{z s}$ values for conventional AFMs are typically larger than $100 \mathrm{fm} / \sqrt{\mathrm{Hz}}$, giving a more than $64 \%$ increase in the noise in FM-AFM. Therefore, the performance of dynamic-mode AFM in liquid is not necessarily limited only by the $\mathrm{Q}$ factor but is often influenced by the noise from the cantilever deflection sensor as well.

In 2005, Fukuma et al. presented the design of a low-noise optical beam deflection sensor with $n_{z s}$ values of $17 \mathrm{fm} / \sqrt{\mathrm{Hz}}$ in air and $40 \mathrm{fm} / \sqrt{\mathrm{Hz}}$ in liquid ${ }^{22)}$ and obtained true atomic- and molecular-resolution images in liquid. ${ }^{24)}$ The major improvement was brought about by the use of laser power modulation at $300 \mathrm{MHz}$ to suppress optical feedback and optical interference noises. ${ }^{22)}$ In 2006, Fukuma et al. made a further improvement by refining the optical design and achieved $n_{z s}$ values of $5.7 \mathrm{fm} / \sqrt{\mathrm{Hz}}$ in air and $7.3 \mathrm{fm} / \sqrt{\mathrm{Hz}}$ in liquid. ${ }^{25)}$ Now, the design is further improved and the present sensor has an $n_{z s}$ value of $4.7 \mathrm{fm} / \sqrt{\mathrm{Hz}}$ in liquid. ${ }^{32)}$ Such an ultralow-noise deflection sensor allows us to obtain the optimal noise performance limited only by cantilever thermal vibration.

As discussed above, true atomic-resolution imaging in liquid with dynamic-mode AFM requires the use of a stiff cantilever and a small oscillation amplitude, which in turn requires a low-noise cantilever deflection sensor. These three factors are the major reasons for the 
dramatic improvements of FM-AFM performance in liquid.

\section{Biological Applications}

In 1993, Ohnesorge and Binnig reported the first true atomic-resolution images obtained in liquid using contact-mode AFM. ${ }^{33)}$ The technique has also been applied to the high-resolution imaging of biological systems such as membrane proteins ${ }^{16)}$ and lipid bilayers. ${ }^{34)}$ Through these previous works, contact-mode AFM has been established as a high-resolution imaging tool at solid/liquid interfaces. In 2005, Fukuma et al. introduced FM-AFM for obtaining true atomic-resolution images in liquid. ${ }^{24)}$ Since there was already an established method (i.e., contact-mode AFM), it was very important to experimentally demonstrate the advantages of FM-AFM in biological research. Compared with contact-mode AFM, FM-AFM has three major advantages in imaging applications, which include the precise control of the vertical tip position at atomic-scale contact/noncontact regimes, high spatial resolution, and capability of imaging isolated molecules. In the following sections, biological applications of FM-AFM highlighting these three major advantages are described. ${ }^{26-28)}$

\subsection{Hydration layers}

In contact-mode AFM, a relatively soft cantilever $(k<0.1 \mathrm{~N} / \mathrm{m})$ must be used to obtain sufficiently high force sensitivity, which hinders the precise control of the vertical tip position at atomic-scale contact/noncontact regimes owing to the jump-to-contact. In the case of liquid-environment applications, it is possible to modify the interaction potential by adding salts to prevent the jump-to-contact; however, this can limit the application range of contact-mode AFM in biology. In addition, soft cantilevers suffer from relatively large thermal fluctuation, which limits the optimal accuracy of the control of the vertical tip position. In contact-mode AFM, the dc deflection of a cantilever is detected and used for the tip-sample distance regulation so that the dc drift of a deflection signal directly results in the dc drift of the vertical tip position and loading force during imaging. In FM-AFM, the high-stiffness cantilever prevents the jump-to-contact and suppresses thermal fluctuation. In addition, the dc drift of a deflection signal hardly influences the $\Delta f$ signal, so that the vertical tip position is stably controlled. This advantage of FM-AFM is highlighted in the following example. ${ }^{26)}$

Water molecules adjacent to biological membranes can potentially play an important role in biological processes, particularly when they form hydration layers. If such hydration layers exist, they should modify the local interaction potential at the water/lipid interface, which can significantly influence the function of membrane proteins and molecular transport across the membranes. FM-AFM has a unique capability of measuring local interaction potentials with a nanometer-scale cross section, using a sharp tip as a force probe. This capability is ideal for directly probing the interaction forces that nanoscale objects (e.g., proteins and solvated ions) experience as they approach a membrane surface. 
Figure 2 shows an example of such applications: a $\Delta f$ vs distance curve measured on a dipalmitoylphosphatidylcholine (DPPC) bilayer supported by mica in phosphate buffered saline (PBS) solution. ${ }^{26)}$ The $\Delta f$ vs distance curves obtained on the DPPC bilayer typically show an oscillatory force profile with one or two peaks. The separation of the two peaks is $0.28 \pm 0.05 \mathrm{~nm}$ on average. This distance corresponds to the size of a water molecule, suggesting that the oscillatory force profile originates from the sequential removal of ordered water molecules.

Tip-sample distance feedback regulation in liquid-environment FM-AFM is performed based on the assumption that $\Delta f$ increases with decreasing tip-sample distance, such as in the force branches indicated by arrows (i) - (iii) in Fig. 2(b). Accordingly, if the $\Delta f$ vs distance curve shows an oscillatory profile, multiple feedback positions can exist for a single $\Delta f$ setpoint as indicated by the circles in Fig. 2(b). Therefore, the tip can spontaneously jump between these positions even if the setpoint is unchanged.

Figure 3 shows an example of such spontaneous jumps during FM-AFM imaging of the DPPC bilayer in PBS solution. ${ }^{26)}$ In the image shown in Fig. 3(a), the tip is scanned from the lowest terrace (terrace 1), which corresponds to the interface between the lipid headgroups and solution. As the imaging progresses, the tip spontaneously jumps twice. Terrace 1 shows a highly ordered arrangement of bright spots separated by $0.50 \pm 0.05 \mathrm{~nm}$ along the stripes. The first jump of $0.26 \mathrm{~nm}$ (terrace 1 to 2 ) results in subtle changes to the image although discrete corrugations are still observed. The second jump of $0.23 \mathrm{~nm}$ (terrace 2 to 3 ) results in a less-ordered contrast, although the image still shows molecular-scale corrugations. The heights of the spontaneous jumps $(0.29 \pm 0.06 \mathrm{~nm})$ agree well with the size of a water molecule and the peak distance of the oscillatory force profile $(0.28 \pm 0.05 \mathrm{~nm})$. This result confirms that the tip jumps between water layers and the individual layers are imaged with molecular-scale corrugations of the lipid headgroups. The result shown here highlights the excellent control of the vertical tip position in FM-AFM, which makes it possible to regulate the tip position even on individual hydration layers weakly interacting with the surface.

\subsection{Lipid-ion network}

In contact-mode AFM, long- and short-range interaction forces are detected with the same sensitivity. In FM-AFM, by reducing the cantilever oscillation amplitude, sensitivity to the short-range interaction force is enhanced while that to the long-range interaction force is reduced. ${ }^{30)}$ Thus, FM-AFM using a small oscillation amplitude should give a higher spatial resolution than contact-mode AFM. This advantage is highlighted by the following example showing the unprecedented spatial resolution of $90 \mathrm{pm}$ in liquid. ${ }^{27)}$

Under physiological conditions, biological membranes are surrounded by an electrolytic solution containing various metal cations. The effect of these cations on membrane structure and stability has been studied intensively using model lipid bilayers. The addition of 
salts can trigger lipid bilayer phase separation, ${ }^{35-37)}$ vesicle aggregation, and vesicle fusion processes. ${ }^{38,39)}$ The striking effect of the ions on membrane structure has highlighted the importance of lipid-ion interactions in biological processes. To date, spectroscopy experiments have revealed that metal cations specifically interact with negatively charged moieties of the lipid headgroups. ${ }^{40-43)}$ These experiments, together with theoretical simulations, ${ }^{44)}$ support the idea that individual ions may interact with multiple headgroups to form complex "lipid-ion networks." This concept has been used to explain the previously observed effects of the ions, such as the enhancement of the mechanical strength of membranes ${ }^{45)}$ and the reduction of the mobility of the lipid molecules therein. ${ }^{46)}$ However, it has been very difficult to experimentally investigate such lipid-ion networks owing to the lack of a viable method for visualizing local lipid-ion interactions with Angstrom resolution. FM-AFM is an ideal tool for this purpose, allowing direct investigation of the local lipid-ion networks formed at water/lipid interfaces.

Figure 4 shows sequential FM-AFM images taken on the DPPC bilayer in PBS solution. The images were taken at the interface between the primary hydration layer and the lipid headgroups. The images show hexagonally packed surface groups separated by approximately $0.5 \mathrm{~nm}$. Each surface group consists of two oval-shaped subunits separated by $0.1-0.3 \mathrm{~nm}$. From detailed analyses of the FM-AFM images and the simulation results previously reported, it was concluded that the observed subnanometer-scale contrasts of the FM-AFM images represent the spatial distribution of mobile ions interacting with the lipid headgroups. The pair of subunits correspond to the enhanced distribution of $\mathrm{Na}^{+}$at the negatively charged oxygen atoms in the phosphatidylcholine (PC) headgroups.

The sequential images reveal that some of the surface groups change their configurations upon the formation or disappearance of lipid-ion networks. For example, subunit 1 (S1) is not paired with another subunit in Fig. 4(a), while a pair of subunits 3 (S3) and 4 (S4) appears in the next image [Fig. 4(b)] but with a darker contrast than the other subunits, suggesting the lower height of this headgroup. It is likely that this particular headgroup was temporarily at an irregular tilt angle due to the interaction between subunits 1 and 2 against the slight height difference when the first image was taken.

The observed structural changes owing to the formation and disappearance of the lipid-ion networks suggest that the negatively charged phosphate groups share the positive charge of cations, by which an attractive electrostatic force is exerted on all the headgroups involved in the network. The attractive interaction force mediated through such complex lipid-ion networks should bind the headgroups together and enhance the global mechanical strength of the membrane. In fact, it was reported that the addition of metal cations increases the mechanical strength of the DPPC bilayer. ${ }^{45)}$ The FM-AFM images presented here reveal the submolecular-scale origin of such an effect of ions on the mechanical properties of the biological membrane. 
If we define "spatial resolution" as the closest distance between two features resolved in one image, it is approximately $90 \mathrm{pm}$ in this experiment. ${ }^{27)}$ This is the highest resolution obtained in liquid so far, which highlights the improved spatial resolution of FM-AFM compared with conventional AFM techniques.

\subsection{Amyloid fibrils}

In contact-mode AFM, scanning a tip in contact with the surface causes a large lateral friction force. This has hindered nondestructive imaging of isolated molecules or macromolecular assemblies weakly bound to a substrate. As the vertical motion of the cantilever in dynamic-mode AFM markedly reduces the lateral friction force, FM-AFM is capable of imaging molecular-scale surface structures of such isolated molecules and molecular assemblies. This advantage was demonstrated by imaging amyloid fibrils in PBS solution as described in the following section. ${ }^{28)}$

Amyloid fibrils are formed from a variety of proteins that are normally soluble in aqueous solution. ${ }^{47,48)}$ Under certain conditions, the precursor proteins misfold to form amyloid fibrils, which are typically $10-30 \mathrm{~nm}$ in diameter and up to several micrometers in length, as shown in Fig. 5(a). The fibrils are insoluble $\beta$-sheet-rich structures that can form aggregates known as amyloid deposits in tissues and organs. These deposits are found in a range of neurodegenerative diseases such as Alzheimer's, Parkinson's, and Huntington's diseases. ${ }^{47}$ ) Understanding the structure of amyloid fibrils is essential for elucidating the nucleation and kinetics of fibrillation in relation to the pathogenic pathway of amyloidoses.

Figures 5(a) and 5(b) show FM-AFM images of amyloid fibrils formed from the islet amyloid polypeptide (IAPP) deposited on mica in PBS solution. IAPP fibrils are associated with type-2 diabetes when deposited in pancreatic islets. ${ }^{49)}$ The image shows finely striped features perpendicular to the fibril axis. The distance between adjacent stripes is approximately 0.5 nm. A previous X-ray diffraction study suggested the existence of a periodic structure along IAPP fibril axes with a characteristic spacing of $0.47 \mathrm{~nm} .{ }^{50)}$ Since this periodicity corresponds to hydrogen bond spacing between the $\beta$-strands [Fig. 5(c)], the periodic structure was attributed to the alignment of the $\beta$-strands perpendicular to the fibril axis. ${ }^{50)}$ The fine stripes observed in the FM-AFM image have comparable spacing aligned perpendicular to the fibril axis. Therefore, the striped features are attributed to the $\beta$-strands of IAPP fibrils.

The images shown here are the first subnanometer-resolution FM-AFM images of isolated biomolecules obtained in liquid, The results demonstrate the possibility of FM-AFM to visualize individual $\beta$-strands in real space in a physiologically relevant liquid environment.

\section{Future Prospects}

The performance of liquid-environment FM-AFM has markedly advanced over the last ten years, which has enabled subnanometer-resolution imaging with a piconewton-order loading 
force at a solid/liquid interface. The biological applications shown here demonstrate that FM-AFM is capable of probing and visualizing the local interactions between biomolecules and the surrounding physiological environment (i.e., water molecules and ions) as well as the structures of biomolecules themselves.

There are still technical issues and challenges to be overcome to make FM-AFM more useful in practical studies of biological issues. The insufficient operation speed of FM-AFM is one of the most serious problems. The improvement of operation speed is important not only for visualizing dynamic processes but also for the high-resolution imaging of large areas containing inhomogeneous complexes and also nondestructive high-resolution imaging of large isolated biomolecules. These applications may not require a high frame rate but do require a high feedback bandwidth. To date, FM-AFM has mainly been used in UHV, where the majority of applications do not require a high feedback bandwidth. Thus, there still remains considerable room for improvement and significant progress is expected in the near future.

Another important issue is the three-dimensional extent of the solid/liquid interface. As shown in Fig. 3, multiple feedback points can exist at the solid/liquid interface owing to the oscillatory force profiles presented by layers of solvent molecules. Although this may be a useful tool for visualization of individual hydration layers, it can also make it difficult to control the vertical tip position. This is not a simple technical issue as the solid/liquid interface is inherently three-dimensional. This issue may be solved by employing the threedimensional imaging technique developed for applications in UHV at low temperature, ${ }^{51}$ ) together with the feedforward drift compensation technique developed for atom manipulation at room temperature. ${ }^{52)}$

There are also many application areas that have not yet been explored fully, such as biology and electrochemistry. So far, the latter has not been explored at all by FM-AFM. This is partly because of the existence of electrochemical STM. However, there is emerging interest in the electrochemical processes of organic and biological molecules in relation to their applications to organic- and bioelectronics. Since these nonconductive molecules cannot be imaged by STM, FM-AFM is expected to be a powerful tool in such application areas.

\section{Acknowledgement}

This research was supported by PRESTO, Japan Science and Technology Agency. 


\section{References}

1) T. R. Albrecht, P. Grütter, D. Horne, and D. Ruger: J. Appl. Phys. 69 (1991) 668.

2) Noncontact Atomic Force Microscopy (Nanoscience and Technology), ed. S. Morita, R. Wiesendanger, and E. Meyer (Springer, Heidelberg 1992).

3) F. J. Giessibl: Science 267 (1995) 68.

4) S. Kitamura and M. Iwatsuki: Jpn. J. Appl. Phys. 34 (1995) L145.

5) S. Orisaka, T. Minobe, T. Uchihashi, Y. Sugawara, and S. Morita: Appl. Surf. Sci. 140 (1999) 243.

6) Ch. Loppacher, M. Bammerlin, M. Guggisberg, F. Battiston, R. Bennewitz, S. Rast, A. Baratoff, E. Meyer, and H.-J. Güntherodt: Appl. Surf. Sci. 140 (1999) 287.

7) K. Fukui, H. Onishi, and Y. Iwasawa: Phys. Rev. Lett. 79 (1997) 4202.

8) M. Bammerlin, R. Lüthi, E. Meyer, A. Baratoff, J. Lü, M. Guggisberg, C. Gerber, L. Howald, and H.-J. Güntherodt: Appl. Phys. A 66 (1998) S293.

9) K. Fukui, H. Onishi, and Y. Iwasawa: Chem. Phys. Lett. 280 (1997) 296.

10) S. Kitamura and M. Iwatsuki: Appl. Phys. Lett. 72 (1998) 3154.

11) K. Kobayashi, H. Yamada, and K. Matsushige: Appl. Phys. Lett. 81 (2002) 2629.

12) U. Kaiser, A. Schwarz, and R. Wiesendanger: Nature 446 (2007) 522.

13) Y. Sugimoto, M. Abe, S. Hirayama, N. Oyabu, O. Custance, and S. Morita: Nat. Mater. $4(2005) 156$.

14) F. J. Giessibl, S. Hembacher, H. Bielefeldt, and J. Mannhart: Science 289 (2000) 422.

15) M. Ternes, Ch. P. Lutz, C. F. Hirjibehedin, F. J. Giessibl, and A. J. Heinrich: Science 319 (2008) 1066.

16) D. J. Müller, F. A. Schabert, G. Buldt, and A. Engel: Biophys. J. 68 (1995) 1681.

17) S. P. Jarvis, T. Uchihashi, T. Ishida, H. Tokumoto, and Y. Nakayama: J. Phys. Chem. B 104 (2000) 6091.

18) K. Kobayashi, H. Yamada, H. Itoh, T. Horiuchi, and K. Matsushige: Rev. Sci. Instrum. 72 (2001) 4383.

19) K. Kobayashi, H. Yamada, and K. Matsushige: Appl. Surf. Sci. 188 (2002) 430.

20) T. Okajima, H. Sekiguchi, H. Arakawa, and A. Ikai: Appl. Surf. Sci. 210 (2003) 68.

21) H. Sekiguchi, T. Okajima, H. Arakawa, S. Maeda, A. Takashima, and A. Ikai: Appl. Surf. Sci. 210 (2003) 61.

22) T. Fukuma, M. Kimura, K. Kobayashi, K. Matsushige, and H. Yamada: Rev. Sci. Instrum. 76 (2005) 053704. 
23) T. Fukuma, T. Ichii, K. Kobayashi, H. Yamada, and K. Matsushige: Appl. Phys. Lett. 86 (2005) 034103.

24) T. Fukuma, K. Kobayashi, K. Matsushige, and H. Yamada: Appl. Phys. Lett. 87 (2005) 034101.

25) T. Fukuma and S. P. Jarvis: Rev. Sci. Instrum. 77 (2006) 043701.

26) T. Fukuma, M. J. Higgins, and S. P. Jarvis: Biophys. J. 92 (2007) 3603.

27) T. Fukuma, M. J. Higgins, and S. P. Jarvis: Phys. Rev. Lett. 98 (2007) 106101.

28) T. Fukuma, A. S. Mostaert, and S. P. Jarvis: Nanotechnology 19 (2007) 384010.

29) T. Fukuma, K. Kobayashi, H. Yamada, and K. Matsushige: Rev. Sci. Instrum. 75 (2004) 4589 .

30) F. J. Giessibl, H. Bielefeldt, S. Hembacher, and J. Mannhart: Appl. Surf. Sci. 140 (1999) 352.

31) F. J. Giessibl: Appl. Phys. Lett. 76 (2000) 1470.

32) T. Fukuma: Rev. Sci. Instrum. 89 (2009) 023707.

33) F. Ohnesorge and G. Binnig: Science 260 (1993) 1451.

34) A. L. Weisenhorn, M. Egger, F. Ohnesorge, S. A. C. Gould, S.-P. Heyn, H. G. Hansma, R. L. Sinsheimer, H. E. Gaub, and P. K. Hansma: Langmuir 7 (1991) 8.

35) M. Rappolt, G. Pabst, H. Amenitsch, and P. Laggner: Colloids Surf. A 183 (2001) 171.

36) M. Ross, C. Steinen, H. J. Galla, and A. Janshoff: Langmuir 17 (2001) 2437.

37) J. T. Groves, S. G. Boxer, and H. M. McConnell: J. Phys. Chem. B 104 (2000) 11409.

38) S. Ohki, N. Düzgüneş, and K. Leonards: Biochemistry 21 (1982) 2127.

39) S. Ohki and K. Arnold: Colloids Surf. B 18 (2000) 83.

40) L. Herbette, C. A. Napolitano, and R. V. McDaniel: Biophys. J. 46 (1984) 677.

41) C. Altenbach and J. Seelig: Biochemistry 23 (1984) 3913.

42) H. Binder and O. Zschornig: Chem. Phys. Lipids 115 (2002) 39.

43) T. R. Hermann, A. R. Jayaweera, and A. E. Shamoo: Biochemistry 25 (1986) 5834.

44) M. L. Berkowitz, D. L. Bostick, and S. Pandit: Chem. Rev. 106 (2006) 1527.

45) S. Garcia-Manyes, G. Oncins, and F. Sanz: Biophys. J. 89 (2005) 1812.

46) R. A. Böckmann, A. Hac, T. Heimburg, and H. Grubmuller: Biophys. J. 85 (2003) 1647.

47) P. Westermark, M. D. Benson, J. N. Buxbaum, A. S. Cohen, S. I. B. Frangione, C. L. Masters, G. Merlini, M. J. Saraiva, and J. D. Sipe: Amyloid 12 (2005) 1.

48) P. Westermark: FEBS J. 272 (2005) 5942. 
Jpn. J. Appl. Phys.

49) P. Westermark, C. Wernstedt, E. Wilander, and D. W. Hayden: Proc. Natl. Acad. Sci. U.S.A. 84 (1987) 3881.

50) O. S. Makin and L. C. Serpell: J. Mol. Biol. 335 (2004) 1279.

51) H. Hölscher, S. M. Langkat, A. Schwarz, and R. Wiesendanger: Appl. Phys. Lett. 81 (2002) 4428.

52) M. Abe, Y. Sugimoto, T. Namikawa, K. Morita, N. Oyabu, and S. Morita: Appl. Phys. Lett. 90 (2007) 203103. 


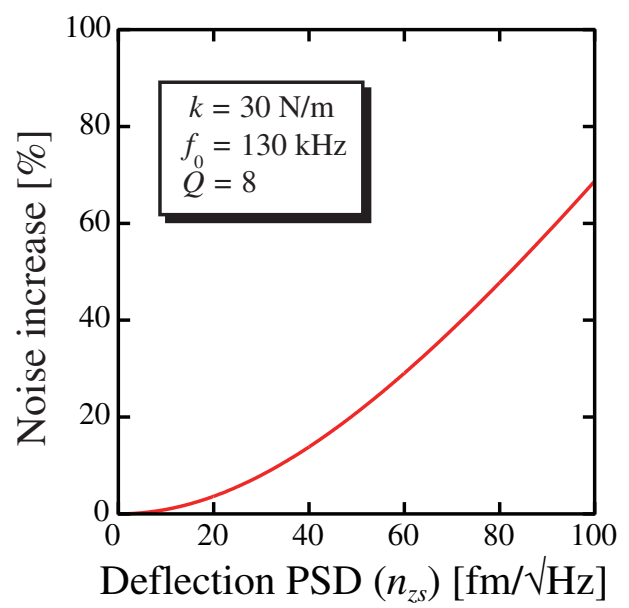

Fig. 1. (Color online). Increase in deflection PSD at $f_{0}$ owing to $n_{z s}$.
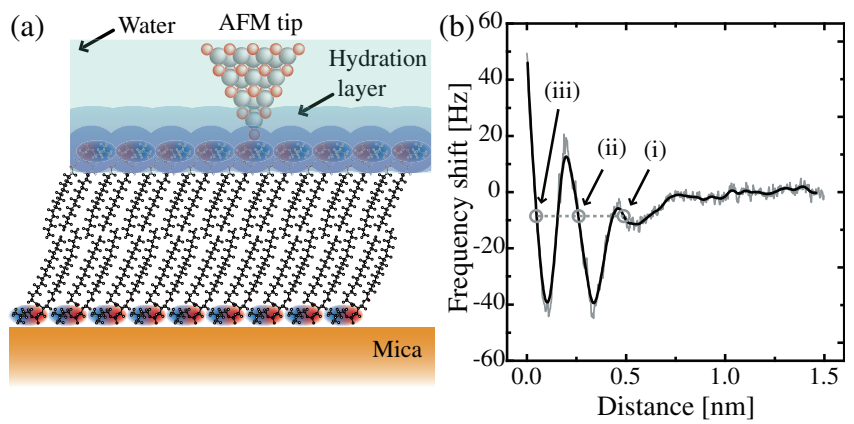

Fig. 2. (Color online). (a) Schematic model of a DPPC bilayer formed on mica investigated by AFM in liquid. (b) $\Delta f$ vs distance curve measured on the DPPC bilayer in PBS solution, showing oscillatory profile with two peaks. The smoothed line (solid) is obtained by averaging the raw data (shaded) over the distance range of $\pm 0.02 \mathrm{~nm}$ from each data point. (Reused with permission from Fukuma et al. ${ }^{26)}$ Copyright 2007, Biophysical Society). 

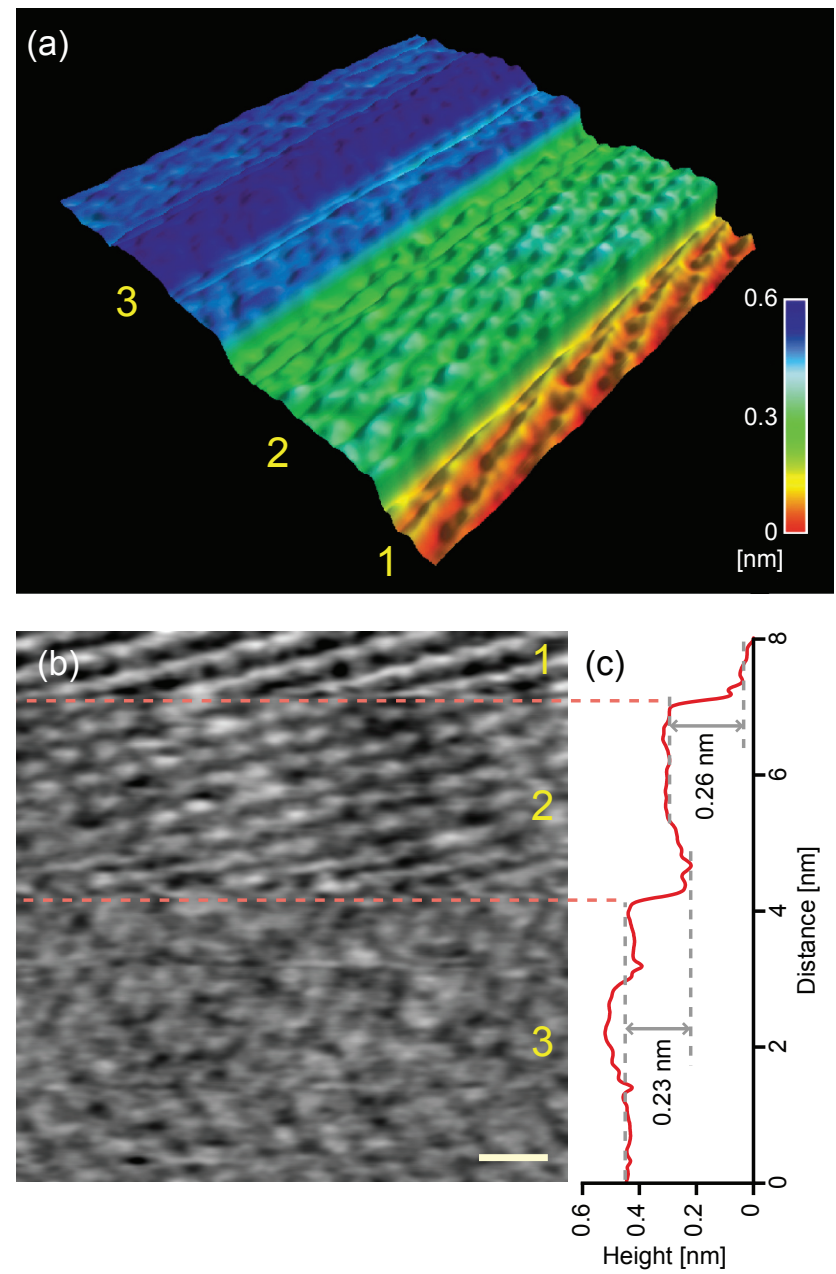

Fig. 3. (Color online). (a) FM-AFM image of the DPPC bilayer in PBS solution showing spontaneous jumps during imaging. Scan size: $8 \times 8 \mathrm{~nm}^{2}$. Tip velocity: $120 \mathrm{~nm} / \mathrm{s}$. Imaging speed: $85 \mathrm{~s} /$ frame. (b) Line-by-line flattened image of (a). Scale bar: $1 \mathrm{~nm}$. Height range: $0.1 \mathrm{~nm}$ (black to white). Fast and slow scan directions: left to right and top to bottom. The regions indicated by the numbers 1 , 2 , and 3 respectively correspond to terraces 1,2 , and 3 in (a). (c) Line-averaged height profile of (b) plotted along the slow scan direction. (Reused with permission from Fukuma et al. ${ }^{26)}$ Copyright 2007, Biophysical Society.)
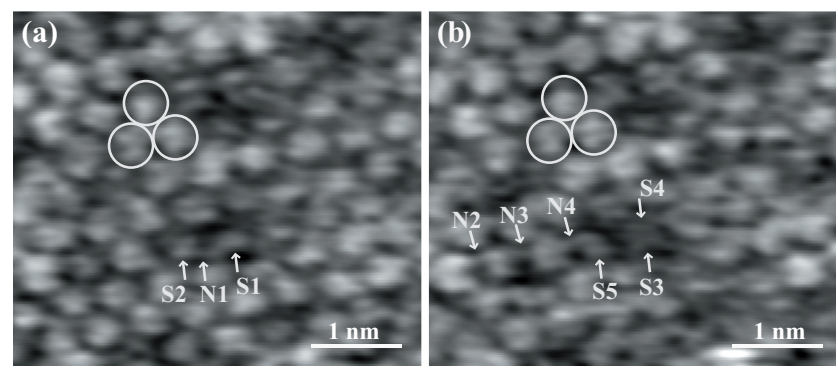

Fig. 4. Sequential FM-AFM images of the same area of the DPPC bilayer in PBS solution. Height range: $0.1 \mathrm{~nm}$. Tip velocity: $120 \mathrm{~nm} / \mathrm{s}$. Imaging speed: $85 \mathrm{~s} /$ image. (Reused with permission from Fukuma et al. ${ }^{27)}$ Copyright 2007, American Physical Society.) 

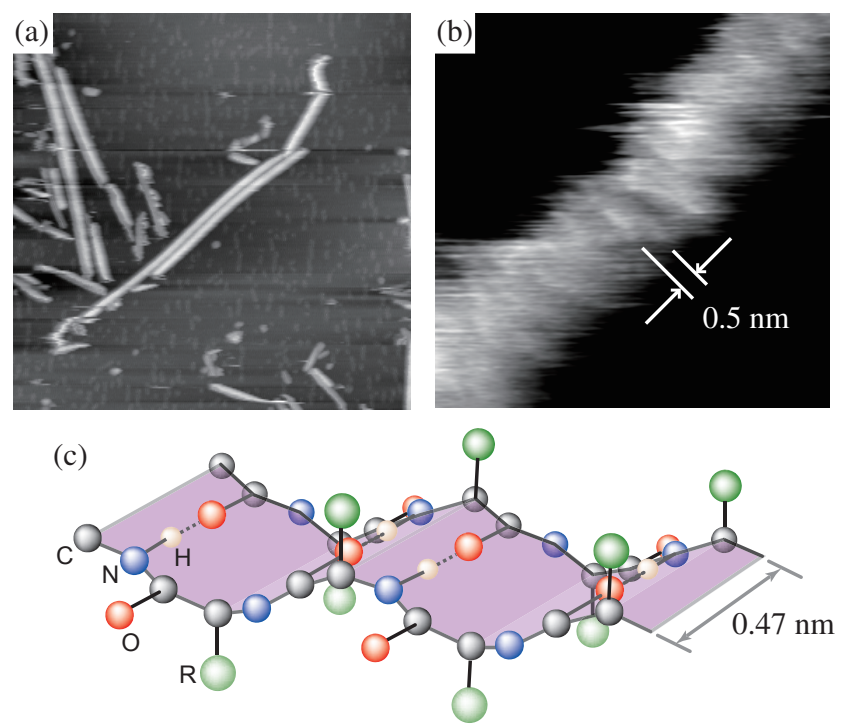

Fig. 5. (Color online). FM-AFM images of IAPP fibrils on mica in PBS solution (a) $800 \times 800 \mathrm{~nm}^{2}$, $\Delta f=-55 \mathrm{~Hz}$, tip velocity: $1 \mu \mathrm{m} / \mathrm{s}$. (b) $10 \times 10 \mathrm{~nm}^{2}, \Delta f=+50 \mathrm{~Hz}$, tip velocity: $195 \mathrm{~nm} / \mathrm{s}$. (c) Schematic model of the $\beta$-strands. (Reused with permission from Fukuma et al. ${ }^{28}$ ) Copyright 2008, Institute of Physics.) 